\title{
EP-155
}

\section{Amylase level after pancreaticoduodenectomy in predicting postoperative pancreatic fistula}

\author{
Kwangyeol PAIK*, Ji Seon OH, Eung Kook KIM, Seugmin LEE
}

HBP Surgery, Yeouido St. Mary's Hospital, The Catholic University of Korea, Seoul, Korea

Introduction: It is new clinical interest higher serum amylase level with pancreatitis after pan-creaticoduodenectomy (PD) correlates with postoperative pancreatic fistula (POPF). Nevertheless, its evidence and study were scarce. We aimed to investigate correlation of serum amylase level immediate after PD and POPF occurrence.

Methods: Of 163 patients who underwent PD at between January 2007 and December 2019, retrospective analysis was conducted to identify risk factors including serum amylase level immediate after PD for POPF occurrence.

Results: Overall incidence of POPF (25/163) was $15.3 \%$. The patients occurred a POPF had significantly higher level of serum amylase on POD 0 compared to in whom without a POPF (414 vs. 253, $p<0.001)$. In univariate analysis, ASA classification, post pancreatectomy acute pancreatitis (POAP, serum amylase on POD $0>285$ IU/L) and Fistula Risk Grade were correlated with POPF occurrence. In multivariable analysis, Fistula risk grade and POAP were significantly associated with developing POPF.

Conclusions: In patients with higher serum amylase (> 285 IU/L) on POD 0 with higher fistula risk grade,comprehensive management to achieve mitigation of POPF is important. 